\author{
Research Article
}

Jonathan Peter Merrison*

\title{
Predictive model linking super-rotation, magnetospheric generation and atmospheric heating
}

https://doi.org/10.1515/astro-2020-0025

Received Sep 01, 2020; accepted Dec 03, 2020

\begin{abstract}
This work applies a previously suggested model of gravitational field propagation to various planetary bodies within the solar system. Primarily the goal has been to critically test the validity of this model by identifying observations which are in direct conflict with it. Specifically this model predicts a Doppler shift in gravitational acceleration (gD). Applying the model to the planets and the Sun $g D$ acts to increase planetary spin, opposing various sources of drag. The model is seen not to be in conflict with a wide variety of observed parameters which have been treated here and is shown to quantitatively account for several observed phenomena previously thought to be unrelated and which have been difficult to explain conventionally. These phenomena include the internal heat generation and magnetospheric generation within the gas giants as well as super rotation which seen in most planetary atmospheres as well as the Sun as differential rotation. This model for the first time provides a quantitative prediction of the low internal heat generation seen in Uranus compared to Neptune. It also provides a novel mechanism for solar coronal heating, thermospheric heating in the gas giants and the correlation between climate and magnetosphere observed on Earth.
\end{abstract}

Keywords: planetology, astronomy, gravity

\section{Introduction}

In previous work a novel model was presented with an alternative approach for applying relativity to gravitational propagation, one which is analogous to and compatible with electromagnetic and other field theories (Merrison 2016). A consequence of this treatment of gravity is that the (relative) velocity of a gravitating mass will affect the observed gravitational field. Specifically an observer will experience an enhanced gravitational field from a body moving towards it and a reduced gravitational field from one moving away from it. Here one should apply a relativistic Doppler shift to gravitational fields Specifically a gravitating body moving towards an observer will gravitate more (have an enhanced observed gravitational field) and one moving away will have a reduced gravitational field. Here one must apply a relativistic Doppler shift to gravitational fields. This velocity induced Doppler shift in $g(g D \approx g . v / c)$ leads to a modified gravitational field given by $g=g \cdot y \cdot(1+v \cdot \cos (\theta) / c)$, where $g$ is the gravitational ac-

Corresponding Author: Jonathan Peter Merrison: Institute for Physics and Astronomy, Aarhus University, Denmark; Email: merrison@phys.au.dk

ə Open Access. @ 2020 J. P. Merrison, published by De Gruyter. (c)) BY License celeration, $\mathrm{v}$ is the relative velocity, $\theta$ is the angle between velocity and $\mathrm{g}$ vectors and $\mathrm{c}$ is the speed of light. The time dilation term $y$ is typically small compared to that of the Doppler effect. Surprisingly no direct laboratory or observational evidence has been identified which is contradictory to the existence of gD. This previous work focused on the effects on dense/massive bodies on galactic scales. Consideration was also made of the effects on spacecraft fly-bys as well as solar spin on planetary orbits. In the case of the effect of a spinning Sun the effect of gD was seen to lead to a systematic shift in the center of gravity (a shift in the gravitational barycenter) and the generation of a small perihelion (apsidal) precession. For Mercury the value of this orbital precession is smaller than current measurement uncertainties. Similarly observed flybys have been inconclusive. Planetary spin (rotation) was briefly considered though only to the extent of comparing the magnitude of $g D$ to that of tidal gradients (dg/dr). For both Earth and Jupiter $g D$ was seen to be significantly smaller than $\mathrm{dg} / \mathrm{dr}$, though in the case of Jupiter they were of the same order of magnitude.

In the present work a more in-depth analysis will be carried out of the predicted effects of gravitational Doppler shifts (gD) which result from the spin for various solar system bodies (planets, moons, asteroids and the Sun) in the 
gravitational fields generated by either their satellites or their primary. This will involve modelling and performing order of magnitude calculations of $g D$ and comparing these with the other physical factors such as tidal drag, atmospheric drag and magnetospheric drag. Predictions will be quantitatively compared to observations. A series of observational phenomena will be considered, these are outlined below. A primary goal here is to establish whether observations within the solar system directly contradict predictions of this model (i.e. the gD effect). However, several of the observed phenomena are currently poorly described or have no satisfactory explanation through conventional models.

\subsection{Super rotation}

This is a prograde differential rotation (increased equatorial rotation, increasing at higher altitude) it is seen in essentially all planetary atmospheres as well as in the convection zone of the Sun. In the case of the Sun there is no accepted explanation for this effect, which is conventionally termed solar differential rotation (Lund et al. 2014). In the case of some planetary atmospheres, for example Venus, which has an extremely powerful super rotation, modelling still struggles to empirically reproduce super rotation, with several competing / conflicting models (Read and Lebonnois 2018; Rishbeth 1971; Zhu 2005, 2006; Lewis et al. 2012; Schneider and Liu 2009; Kundt 1983).

\subsection{Planetary magnetic field generation}

The conventional qualitative model to explain magnetic field generation for example for Jupiter (and other gas giants) is through internal convection (internal heating) driving charge transport. A detailed understanding of the process is lacking (Modisette 1967; Siscoe 2001). The magnetosphere can in principle be an infrastructure through which torque (and power) can be transferred between the inner (subsurface), the atmosphere and the outer most exosphere of planets, moons and the Sun. Although the details of this transfer will not be considered here, $g D$ constitutes a novel source of torque (and power) and can balance various drag factors.

\subsection{Internal heat source of the gas giants}

Observation of IR emissions demonstrate that a significant power (heat) generation source exists within the gas giants (below the visible upper atmosphere) of order $10^{14}-10^{17} \mathrm{~W}$.
The conventional qualitative explanation for this is through instability, specifically continued gravitational collapse, due possibly to a phase change of hydrogen, though this has yet to be demonstrated (Hanel et al. 1983; Li et al. 2018; Hunten and Veverka 1976).

Gas giants are seen to have significant zonal winds, their origin remains controversial, though thought to be related to internal heating. Competing models include those based upon deep thermal convection and others combining convective circulation and a thin stably stratified weather layer (Kong et al. 2018; Chanover 2013).

Note that for the terrestrial planets internal heating is thought to be dominated by radioisotope decay.

\subsection{Low internal heating for Uranus compared to Neptune}

Despite Neptune and Uranus being extremely similar in many respects, the internal heat source of Uranus is around an order of magnitude lower than Neptune. No conventional explanation for this has been found. If magnetic field generation is a result of convective flow then it would be expected to result from internal heating which is abnormally low for Uranus. However Neptune and Uranus generate similar magnetic fields. This anomaly currently has no explanation (Helled et al. 2011).

\subsection{Solar Coronal heating problem}

The extremely high coronal temperature (of order $10^{6} \mathrm{~K}$ ) compared to the photospheric temperature (of order $10^{3} \mathrm{~K}$ ) is conventionally problematic to explain and currently several competing qualitative models exist (Klimchuk 2006; Aschwanden 2001). These include a mechanism involving a large number of small, impulsive heating events called nanoflares and another model involving magnetohydrodynamic (MHD) waves (Ishikawa et al. 2017; Kumar et al. 2006). The power (heat) source which is required to explain the observed Coronal heating varies from $<10 \mathrm{~W} / \mathrm{m}^{2}$ to $>1000 \mathrm{~W} / \mathrm{m}^{2}$ for so called quiet to active regions of the Sun (Aschwanden 2001, 2005; Pandey and Narain 2001; Suess 1999). For the gas giants there is also observed a heating process which elevates significantly their thermospheres ( $400-1000 \mathrm{~K}$ for all gas giants), again there is no definitive explanation for this observation (Hunten and Veverka 1976; Yung et al. 1990). 


\subsection{Spin and orbital stability of the planets and moons}

Generally conventional models of the solar system predict that most orbits and rotational spin are in fact unstable due to tidal drag, though that the degree of instability is low enough for this to be difficult to measure and still allow solar system evolution/survival. The gas giants have relatively high spin velocity and typically several massive (slowly orbiting) satellites, this is also true of the Sun. These bodies are far from stability according to conventional models of tidal drag on the orbit of their satellites. The spin of the Earth and Venus are also assumed to be is unstable, whereas mercury is spin-orbit (tidally) locked and Mars has only low mass moons (Macdonald 1964; Goldreich and Soter 1966).

These various observational properties of the solar system will be treated (quantitatively) with regard to the proposed Doppler shift component gD.

\section{Method}

In this section models will be presented in order to apply the effect of a Doppler shift in gravitation $(g D)$ to bodies spinning within an external gravitational field. This might be termed a differential gravitational Doppler effect. This is relevant to the spin of planets, moons and the Sun with regard to their rotational stability as well as being compared to other rotational drag factors.

Massive planetary bodies (planets, major moons, the Sun) are best modelled by incompressible viscous fluid e.g. a lithosphere or a liquid $\mathrm{H}_{2}$ layer. If they have an atmosphere this would be best modelled by a compressible fluid (gas or plasma). These two cases will be treated separately below both with respect to $\mathrm{gD}$ effects and also tidal drag. In addition to the torque applied by tidal and gD effects many planetary bodies have/generate a magnetic field which also applies significant torque (specifically gas giants and the Sun). Here methods will be presented to perform order of magnitude estimates of these sources of torque as well as estimates of power generation. Additionally stress transfer (torque) between the atmosphere and surface will be considered in the case of for example Venus.

The fundamental difference between the cases for incompressible liquid and compressible gas is that for liquid the lateral acceleration is translated into vertical displacement which can then be dissipated, in principle without causing torque transfer. In the case of gas the lateral accel- eration is translated into lateral compression which maintains (elastic) transfer of torque to the rest of the body.

In the case of frictionless bodies and completely rigid bodies models will be applied, possibly relevant for the case of smaller asteroids.

\subsection{Frictionless and rigid bodies}

The primary effect of applying spin to a body which is being orbited by a satellite is to induce an effective systematic shift in the center of mass i.e. a small offset in the $\mathrm{g}$ vector seen by the satellite. For the satellite this systematic shift in the center of mass would induce a perihelion precession of the orbit. This is typically a minor effect and has been treated previously (Merrison 2016). An example is the case of Mercury which has a perihelion precession with observed magnitude much larger than that predicted by $\mathrm{gD}$.

For a perfectly rigid body spinning in a gravitational field the primary effect of $g D$ torque would be to produce a prograde acceleration, increasing pull on the side of the body travelling towards the source of gravitation and reducing the pull on the side travelling away from the gravitational source compared to the center of mass. Generally an applied torque $(T)$ can be related to a relative rate of increase of the angular velocity $(w)$ of around;

$$
d w / d t=T / I=(5 / 2) \cdot T / M R^{2}
$$

Where $I, M$ and $R$ are the inertia, mass and radius of the body respectively.

Therefore a rigid body with applied $g D$ torque will experience a relative rate of angular velocity increase approximated by:

$$
(d w / d t) / w=(0.4) \cdot(5 / 2) . g o / c=g o / c
$$

The value (0.4) is a geometric factor, go is the external gravitational acceleration. Note that a spin-orbit locked body will experience no relative spin or $g D$ torque.

Planets, planetesimals and moons are not well modelled by a rigid body, but rather by a viscous fluid (incompressible liquid), see section 2.2. Depending on their internal structure some smaller asteroids $(\ll 1 \mathrm{~km})$ or possibly also the inner core of some planets/moons might behave as a rigid body. Asteroids will be discussed further in the results section 3.2.3.

\subsection{Liquid (incompressible fluid $g D$ torque)}

The primary effect of tidal gradients on an incompressible fluid sphere, for example by a massive satellite, is the distortion of the sphere along the direction of the tidal gradient 

a) Incompressible fluid
b) Incompressible fluid
c) Compressible fluid
d) Compressible fluid
tidal torque
gDoppler torque

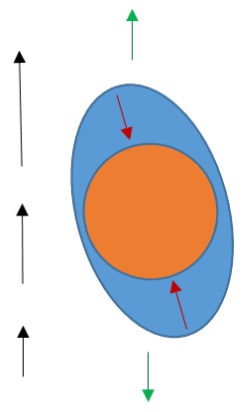
Green arrow tidal stress
Red arrow dissipation
Black line dg/dr

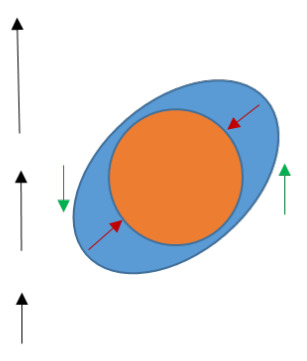
Green arrow gDoppler stress Red arrow dissipation Black line dg/dr
tidal torque

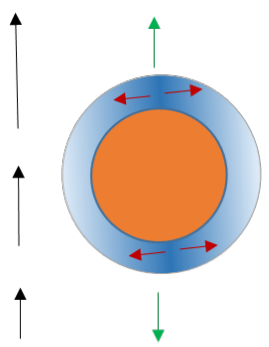
Green arrow gDoppler stress Red arrow dissipation Black line dg/dr
gDoppler torque

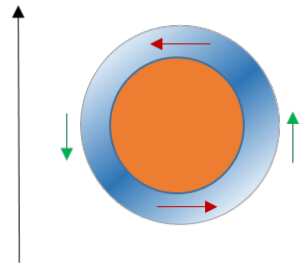
Green arrow gDoppler stress Red arrow dissipation Black line $\mathrm{g}$

Figure 1. a) the conventional model for tidal drag for an incompressible viscous liquid, b) a similar model applied for $g D$ acceleration i.e. Doppler shift of gravitational acceleration in the direction of movement compared to the center of gravity, $g D$ enhanced on the right side of the body and reduced on the left). c) Tidal drag applied to a gaseous compressible fluid, note that it is similar to conventional tidal drag except that in the tidal direction the atmospheric pressure is reduced drawing in gas thus increasing the density (in the dark coloured regions) and causing again a tidal type drag with likely a different lag angle to that of case $1 a$. d) $g D$ acceleration applied to a gaseous compressible fluid (e.g. atmosphere) note that in this case the effect is a first order effect (primarily dependent upon the external $g$ field), note in this case the dark blue regions correspond to increased pressure and density as they are in a compression zone. For the models a)-c) the effect is second order in that it is a result of the generation of mass asymmetry and then dependent upon the gradient in $g$ applied to that mass asymmetry. In all cases the planet/moon rotation is anticlockwise and the imaginary gravitational body is towards the top of the figure. Black arrows indicate the $g$ field (or gradient in $g$ ).

(towards the satellite). Apart from dissipating energy on relaxation this would not cause the application of torque. Tidal torque is however generated by a second order effect in which the tidal distortion is modelled by the generation of a pair of massive bulges. The bulges becomes displaced from the direction of the satellite due to the spin of the planet and a lag in bulge relaxation (see Figure 1a and 1b). A torque is thus generated (gravitational attraction) between the satellite and the bulges. If the spin has a shorter period than the satellite orbit and is spinning in the same plane then this torque will decelerate the spin and accelerate the orbit. If a satellite is spinning in the perpendicular plane to its orbit then there will be no tidal or $\mathrm{gD}$ effects.

The conventional expression for predicting tidal drag makes use of predicting the size of the tidal bulge on for example the planet by a second body say a moon. This is estimated by comparing the gravitational field at the surface of the planet with the stress produced by the moon. This leads to:

$$
\text { Tidal bulge-mass } \approx k \cdot m \cdot\left(R^{3} / r^{3}\right)
$$

where $k$ is a tidal love number, $m$ is the mass of the moon, $R$ is the radius of the planet and $r$ is the distance to the moon. The drag force of the moon on this bulge is then given by $F \sim$ (bulge-mass). $G m R / r^{3}$. The torque of the moon on the planet is then given by the so called lag angle $(\delta$ ) i.e. the angular lag between the direction of the moon and the generated tidal bulge in the planet. The lag is caused by delayed response/dissipation in the planet and can be expressed as a quality factor $Q(\delta=1 / Q)$, see Figure 1 . Thus:

$$
\text { Tidal torque }=T T \approx(k / Q) \cdot G m^{2} \cdot R^{5} / r^{6}
$$

This is only a crude approximation as the $Q$ values are poorly determined. For the terrestrial planets values for lag angle are of order $10^{-1}-10^{-2}$ leading to empirical values of $k / Q$ of order $10^{-2}$. For the gas giants lag angles are thought to be $10^{-3}-10^{-5}$ empirically leading to $k / Q$ of order $10^{-4}$ (Goldreich and Soter 1966).

As with tidal effects the primary effect of $g D$ will be to inflate/deflate the surface. However the mechanism for bulge generation from $g D$ differs from tidal gradients. With $g D$ a lateral (tangential) acceleration rather than vertical one is applied leading to compression/decompression zones, see Figure 1b.

A similar methodology to equations 3 and 4 can be employed to estimate the torque applied due to $g D$; the bulge mass on a planet by a moon can be estimated by comparing the gravitational field at the surface of the planet 
with the gD produced by the moon, giving;

$$
\text { gD bulge-mass } \sim k \cdot m .\left(R^{2} / r^{2}\right) .(v / c)
$$

The drag force of the moon on this bulge is then given by $F \sim$ (bulge-mass). $G m R / r^{3}$. Note that $v=w R$. The torque of the moon on the planet is then given by a lag angle $(1 / Q D)$, however in this case the maximum velocity gradient is seen as the surface travels towards the moon and the minimum Doppler acceleration is seen when the moon is normal to the surface (the inverse of tidal distortion). The maximum Doppler bulge might be expected to be found between the maximum acceleration and maximum relaxation angle, this would not necessarily be related to the tidal $k / Q$ factor and not necessarily a small angle.

$$
g D \text { torque }=T D \sim(k / Q D) \cdot G m^{2} \cdot R^{4} / r^{5} \cdot(v / c)
$$

The empirical parameter $(\mathrm{k} / \mathrm{QD})$ is probably $\ll 1$.

For both tidal and especially for $g D$ the amplitude of the tidal or $g D$ bulge are expected to be small. The torque generated is therefore a second order effect and expected to be relatively inefficient. For tidal drag this efficiency is typically less than $1 \%$ (i.e. the values of $k / Q$ ). Heat generation through dissipation is also a secondary effect and similarly inefficient. It is unclear for the Sun (composed of plasma) whether the liquid layer model can (at any depth) be applied. It has been assumed here that at least the outer $10 \%$ of the Suns radius resembles a compressible fluid layer (see section 2.3).

\subsection{Atmospheres compressible fluid $g D$ torque}

For compressible gas tidal or $g D$ stress effects do not cause a bulge to form. Instead a compression region is produced i.e. a region of the atmosphere in which the pressure is enhanced. This compression is essentially an adiabatic process making it relatively lossless compared to tidal drag/ $g D$ for liquids. For tidal stress there will thus be no primary torque and relatively small secondary torque (due to low lag). For $g D$ however, this process directly (efficiently) converts the gravitational Doppler forces into a tangential wind and therefore a primary torque upon the atmosphere (see Figure 1d). Qualitatively this might explain the superrotation observed in planetary atmospheres and the Sun (assuming plasma acts like a gas). Quantitatively this torque acting upon the entire atmosphere can be estimated;

$$
T D=m^{\prime} \cdot g o \cdot(v / c) \cdot R \cdot(0 \cdot 4) \cdot \cos (t i l t)
$$

The value (0.4) is a geometric term from integrating velocity angle for a spinning sphere. Importantly go is the total $\mathrm{g}$ experienced by the planet (ignoring gradients in $g$ ). For determining $m^{\prime}$ (the effective atmospheric mass) estimates of the atmospheric density and thickness are required. These are essentially empirical parameters although based upon reasonable (order of magnitude) values i.e. around $0.4 \mathrm{~kg} / \mathrm{m}^{3}$ and a depth of around $100 \mathrm{~km}$ for Jupiter and Saturn and around $1000 \mathrm{~km}$ for Uranus and Neptune (see Table A2). Interestingly these assumptions lead to similar atmospheric mass for all of the gas giants. For the Sun the outermost around $14 \%\left(10^{5} \mathrm{~km}\right)$ has been considered.

\subsection{Magnetospheric torque (Jupiter, gas giants, the Sun)}

The conventional explanation for the generation of planetary and stellar magnetospheres is that they are driven by internal heating which leads to convective flow. This process is poorly described. The effects of the Doppler shift of gravity $(g D)$ is to increase the angular momentum of the gas giants and therefore there is the potential for this to drive magnetic field generation by directly affecting the flow of charge carriers within the planet.

The solar wind (and solar magnetosphere) apply drag to the outer parts of planetary magnetospheres, impacting at the bow shock. An estimate for the torque applied to the planetary magnetosphere by the solar wind can be made knowing the relative velocity (vsw), the area of the magneto-shock of the planet $(\mathrm{AB})$ and the solar wind flux or, considering the solar wind as a fluid then the solar wind pressure Psw;

$$
T B=P s w . A B \cdot(R p R B)
$$

Where $A B=\pi \cdot(R p R B)^{2}, \mathrm{Rp}$ and $\mathrm{RB}$ are respectively the planetary and relative bow shock radii, Psw $=$ solar wind pressure at the planet bow shock. Here the solar wind current is assumed to decreases as $1 / r^{2}$. Empirically the solar wind pressure has been taken as around $6 \mathrm{nPa}$ at $1 \mathrm{AU}$ (at Earth) (Richardson et al. 1996), see Table A3. The potential power generation (for frictional solar wind interaction at the bow shock) would be crudely:

$$
d E / d t=P s w \cdot A B \cdot v B
$$

Where $v B$ is the relative solar wind velocity crudely $=$ solar wind velocity $+B$ field velocity $\approx v s w+R B . R p . w$.

In addition to the solar wind another significant source of drag on the magnetosphere could come from plasma loss (injected into the magnetosphere) from the moons, the rings or the planet's atmosphere (thermosphere). A similar 
expression for the applied torque due to plasma loss can be used knowing the mass loss rate $(d m / d t)$ :

$$
T m=(d m / d t) \cdot v B \cdot R B \cdot R p
$$

Estimating the total mass loss from the various sources is challenging. For Jupiter and Saturn it is likely to be $\mathrm{dm} / \mathrm{dt}$ > $1000 \mathrm{~kg} / \mathrm{s}$ (Cowley et al. 2015). Estimates of single moons for Saturn are of order $100 \mathrm{~kg} / \mathrm{s}$ (e.g. for Enceladus) (Holmberg et al. 2016). For the rings latest estimates are up to $10^{4}-10^{5}$ $\mathrm{kg} / \mathrm{s}$ (O'Donoghue et al. 2019). This is based upon the latest estimates for the lifetime of the rings of Saturn (to plasma) of order $10-100$ Myrs and mass of around $2 \cdot 10^{19} \mathrm{~kg}$. Similar large total mass loss might be possible for the outer gas giants. Note also that the high thermospheric temperatures seen for the gas giants might enhance plasma loss from the planets' atmosphere.

\subsection{Atmospheric - surface drag (Venus)}

Torque (and energy) transfer between the atmospheric layer and the liquid layer (surface) can occur through aerodynamic drag. Since Venus has essentially no magnetic field $g D$ torque cannot be balanced by magnetospheric torque, super rotation in the atmosphere might then be expected to increase until aerodynamic drag with the surface balances the $g D$ torque. Surface aerodynamic drag can be estimated from an order of magnitude approximation of the surface shear stress (due to super rotation:

$$
T A=\left(C d \cdot \rho \cdot U s^{2} \cdot A p\right) \cdot R p \cdot(0.4)
$$

$C d=S k i n$ friction coefficient with order of magnitude estimate of 0.001 , Us is the super rotation component of the near surface wind speed, $\rho$ is the surface atmospheric density. Measured Venus surface wind speeds are at least $80 \%$ of the time $>0.1 \mathrm{~m} / \mathrm{s}$ (Yamamoto 2017).

Note that for Earth and Mars there is a relatively large solar flux (of order $1 \mathrm{~kW} / \mathrm{m}^{2}$ ), causing dominant thermospheric winds and therefore difficulty in estimating Us. Venus, however, has a thick cloud layer and therefore only a few $\%$ of this flux at the surface. Titan similarly has a low solar flux. Both Venus and Titan are extreme examples of super rotation.

\subsection{Power balance (atmospheric heating)}

Power generation, for example between various sources of torque $(T)$, can be estimated using equation;

$$
d E / d t=w \cdot d L / d t=w \cdot T
$$

For the gas giants these power sources can be compared to observed internal heat generation. Here internal refers to heat generated below the visible atmosphere (i.e. in the lower atmosphere). This power source might also lead to heat generation in the upper atmosphere (thermosphere or solar corona).

\section{Results and discussion}

In this section the results of order of magnitude calculations of the $g D$ effect will be presented for moons, planets and the Sun, including both their liquid and gaseous layers. A primary goal here is to identify observational parameters which are in direct contradiction with the existence of a Doppler shift in solar system gravitational fields.

Generally the magnitude of $g D$ forces are small with regard to direct measurement, typically at least an order of magnitude smaller than gravitational tidal gradients, see Table A5. The ratio between the amplitude of these effects from tidal gradients $(d g / d r)$ and $g D$ can be determined by comparing equations 2 and 4; (tidal bulge mass) $/(g D$ bulge mass $)=c R / v r$. On Earth direct observations of both liquid layer tidal bulge and atmospheric tidal pressure amplitudes have been made, however estimated $g D$ amplitudes for both the Moon and Sun are more than an order of magnitude lower than these values, specifically around $\times 10^{4}$ and $\times 28$ respectively and unlikely to be discernable.

In the following sections more detailed model results will be presented subdivided by planetary body as well as atmospheric or liquid layers. Details of the estimated parameters and assumptions are presented in the appendix material Tables A1 - A5. The main results are summarized in Table 1.

In most cases not only is it seen that the effects of $g D$ are indeed not contradicted by observation, but that the effect allows improved stability within the solar system. In some cases previously poorly understood phenomena can be quantitatively explained and seemingly unrelated peculiarities linked. These include the ubiquitous phenomenon of super rotation (including the differential rotation in the Sun), the observed internal atmospheric heating of the gas giants, the abnormal internal heating of Uranus and the source of magnetospheric torque / power as well as heating the Suns corona. 
Table 1. A compilation of order of magnitude estimates of; Atmospheric $g D$ torque on gas giants, the Sun and Earth (using equation 7 , Table A2), Magnetospheric torques due to solar wind pressure (equation 8, Table A3, Table 1, columns 1 and 2), power balance for these factors are shown (equation 9 and 12, Table 1, columns 3-5). For the gas giants the power balance is compared to the observed 'internal' heat generation, for the Sun the power balance is compared to the observed 'quiet' coronal heat generation, for the Earth the thermospheric $\left(\mathrm{CO}_{2}\right.$ and NO) IR emission power is taken only (Mlynczak et al. 2016). Torques are estimated for the liquid layers of the planets (lithosphere) including $g D$ acceleration and tidal drag (equations 4 and 6, Table A6, Table 1, columns 6 and 7 ). For the gas giants the tidal $(k / Q T)$ parameter has been taken as $10^{-4}$, for Earth the tidal $(k / Q T)$ parameter has been taken as $10^{-2}$, for $g D$ the $(k / Q D)$ parameter has been taken as $10^{-2}$, see section 3.2.

\begin{tabular}{|c|c|c|c|c|c|c|c|}
\hline \multirow[b]{2}{*}{ Body } & \multicolumn{2}{|c|}{ Atmospheric Torque } & \multicolumn{3}{|c|}{ Atmospheric: power balance } & \multicolumn{2}{|c|}{ Liquid layer Torque } \\
\hline & $\begin{array}{l}g D \text { torque } \mathrm{Nm} \\
\text { (accel) }\end{array}$ & B torque $\mathrm{Nm}$ (drag) & $g D$ power W & $\begin{array}{c}B \text { field power } \\
\text { W }\end{array}$ & $\begin{array}{c}\text { observed heat } \\
\text { W }\end{array}$ & $g D$ torque $\mathrm{Nm}$ & $\begin{array}{c}\text { Tidal Torque } \\
\qquad \mathrm{Nm}\end{array}$ \\
\hline Jupiter & $+0.8 \times 10^{21}$ & $-3 \times 10^{21}$ & $+1 \times 10^{17}$ & $+8.0 \times 10^{17}$ & $-1.1 \times 10^{17}$ & $+5 \times 10^{17}$ & $-9 \times 10^{18}$ \\
\hline Saturn & $+9 \times 10^{19}$ & $-3 \times 10^{18}$ & $+2 \times 10^{16}$ & $+0.1 \times 10^{16}$ & $-2 \times 10^{16}$ & $+2 \times 10^{15}$ & $-2 \times 10^{16}$ \\
\hline Uranus & $+5 \times 10^{17}$ & $-2 \times 10^{16}$ & $+7 \times 10^{13}$ & $+2 \times 10^{13}$ & $-7 \times 10^{13}$ & $+2 \times 10^{13}$ & $-1 \times 10^{15}$ \\
\hline Neptune & $+3 \times 10^{18}$ & $-2 \times 10^{16}$ & $+3 \times 10^{14}$ & $+0.1 \times 10^{14}$ & $-6 \times 10^{14}$ & $+1 \times 10^{14}$ & $-4 \times 10^{15}$ \\
\hline Sun & $+2 \times 10^{24}$ & $-1 \times 10^{24}$ & $+0.7 \times 10^{19}$ & $+0.3 \times 10^{19}$ & $-6 \times 10^{19}(\mathrm{cor})$ & NA & NA \\
\hline Earth & $+1 \times 10^{17}$ & $-0.4 \times 10^{17}$ & $+8 \times 10^{12}$ & $+16 \times 10^{13}$ & $-1 \times 10^{12}(\mathrm{th})$ & $+1 \times 10^{14}$ & $-8 \times 10^{15}$ \\
\hline
\end{tabular}

\subsection{Atmospheric $g D$ acceleration and magnetospheric drag}

In this section the predicted $g D$ acceleration of various atmospheres (including the Suns plamasphere) are quantified and compared to estimated magnetospheric drag. These values are then compared to observational properties such as the magnitude of super rotation, the required torque/power to maintain the magnetosphere, internal heating in the gas giant lower atmosphere, heating of planetary upper atmospheres (thermospheres) or solar coronal heating.

\subsubsection{Gas giants and Earth}

This model for $g D$ applied to the gas giant atmospheres (using equation 7) predicts a large prograde torque, dominated by the gravitational field of the Sun (see Figure 1d). Qualitatively this predicts atmospheric super rotation. However, for the planetary atmosphere to be stable this torque must be balanced by some form of drag. One obvious source of drag is that applied to the magnetosphere by the solar wind (and Suns magnetosphere). Conventionally this magnetospheric torque is not quantitatively described. As shown in Table 1 (columns 1 and 2) rough order of magnitude (ROM) estimates of the $g D$ torque and magnetospheric torque are in good agreement for Jupiter as well as Earth (using equations 7 and 8, Tables 1, A2 and A3).

For the gas giants assuming these forces are balanced through frictional exchange in the lower atmosphere allows the prediction of internal heat generation. For Jupiter again there is order of magnitude agreement between $g D$ power, magnetospheric power and the observed heat power generation (Table 1, columns 1 and 2). Similarly for the remaining three gas giants there is good agreement with the power generated by $g D$ in the atmosphere and the observed internal heat generation for these planets (using equations 12 , 7 and 8 , Table 1 , columns 3-5, Tables A2 and A3). This includes Uranus which has an unusually low internal heat generation unaccounted for by conventional models such as gravitational collapse or a phase change. Here the higher value for the predicted energy generated by $g D$ torque for Neptune compared to Uranus, despite using the same values for average density and atmospheric thickness, is due to a combination of the extraordinarily high tilt of Uranus (reducing the $g D$ of the Sun) and the extraordinarily massive/close Neptunian moon Triton which dominates the $g$ field (see Table A5). Both Uranus and Neptune are unusual in several ways, they have no clear super rotation, they have highly non aligned spin, orbital and magnetic field orientations also Triton is extraordinarily in a retrograde orbit about Neptune. These issues may in fact be related.

Unlike Jupiter for the gas giants Saturn, Uranus and Neptune the $g D$ torques are however not well balanced by magnetospheric drag due to the solar wind estimates (see Table 1 and Table A2). Possible sources of additional magnetospheric drag for these cases might be plasma losses by moon, rings and the atmosphere. By applying equation 10 the required mass loss rate from these various sources in order to balance the predicted $g D$ torque would be of order $10^{4}-10^{5} \mathrm{~kg} / \mathrm{s}$ for Saturn and $10^{2}-10^{3} \mathrm{~kg} / \mathrm{s}$ for Uranus and Neptune. This is in reasonable agreement with upper estimates of mass loss of the rings, moons and atmosphere to plasma (see section 2.4). A more detailed study would be required to resolve this issue. Note that solar wind and 
plasma emissions are extremely variable and reliable mean values are difficult to establish.

If for the gas giants Saturn, Uranus and Neptune the $g D$ torques are unbalanced by magnetospheric drag, the time constant for the instability in angular velocity can be determined using equation 1, (i.e. $d t=w /(d w / d t)$ ), respectively these are $5.10^{10} \mathrm{yrs}, 2.10^{11} \mathrm{yrs}, 2.10^{10} \mathrm{yrs}$. These long time scales (low deceleration) would not be directly observable or significantly decrease stability of the solar system.

It is not clear where the torque exchange between $g D$ acceleration and magnetospheric drag occurs, only that that they appear to be in approximate balance. Exchange could occur anywhere between the exosphere/ionosphere (upper atmosphere) and the atmospheric base (close to the contact region with the liquid surface). Torque exchange in the upper most atmosphere for the gas giants can qualitatively explain the high thermosphere temperatures of these planets which otherwise remains problematic to explain. For the Earth torque exchange between the atmosphere and magnetosphere might explain the observed and otherwise poorly understood correlation between magnetic variations and climate (Courtillot et al. 2007). The power generation from torque exchange in the Earth's upper atmosphere is capable of being partially balanced by thermospheric cooling (Mlynczak et al. 2016), however solar flux effects likely dominate. For Earth (and the other terrestrial planets) internal heating is seen to be dominated by radio-generation (Yung et al. 1990; Hunten and Veverka 1976).

\subsubsection{Sun}

Similarly to the gas giants applying equation 4 to the plasma 'atmosphere' of the Sun (i.e. the upper part of the convection zone including the outer $14 \%$ of the solar radius) predicts a large prograde torque (see Table 1 and Table A2), dominated by the gravitational field of Jupiter (see Figure 1d, see Table A5). Qualitatively this predicts atmospheric super rotation, in this case conventionally termed differential rotation. Again for stability this torque must be balanced by some form of drag and again one obvious source of drag is that applied to the magnetosphere by the solar wind (plasma loss). As shown in Table 1 ROM estimates of the $g D$ torque and magnetospheric torque (equation 8) for the Sun are in good agreement (of order $10^{24} \mathrm{Nm}$ ), strongly supporting its role in the generation of differential rotation in the Sun. Conventionally there is no explanation (qualitative or quantitative) for the origin of the Suns differential rotation i.e. prograde super rotation. However, coupling of convection with solar rotation has been suggested as a possible source for meridional flows (Lund et al. 2014; Miesch and Toomre 2009).

Although due to the high radio-generation of internal power in the Sun power balance cannot be meaningfully carried out for the lower 'atmosphere'. Assuming power balance between $g D$ torque and magnetospheric torque (equations 7, 9 and 12) occurs at the outer solar 'atmosphere' this torque balance provides a novel mechanism for heating of the Corona which is an ongoing problem for conventional models (see section 1.5). The potential power generation of the combined $\mathrm{gD}$ torque and magnetospheric torque integrated over the solar surface is around $7.10^{18} \mathrm{~W}+3.10^{18} \mathrm{~W}=$ $10^{19} \mathrm{~W}$, this is in reasonable order of magnitude agreement with the observed heating of the Corona of around $6.10^{19}$ $\mathrm{W}$ (in a quiet region). A more detailed analysis would be necessary to be conclusive here (see Table 1, A2 and A3).

\subsubsection{Venus (Earth, Mars, Titan, Mercury)}

Venus has a thick atmosphere and significant predicted $g D$ torque (using equation 7, see Table 2 and Table A2). However Venus has (essentially) no magnetosphere, therefore based upon the arguments presented for the gas giants (and the Sun) one would expect that the $g D$ torque upon the atmosphere would be unbalanced by magnetospheric drag and therefore be unstable. There is in fact an extremely powerful super rotation on Venus (of order $100 \mathrm{~m} / \mathrm{s}$ in the upper atmosphere). In this case it may be balanced by aerodynamic drag to the surface. Balance may then occur to tidal drag or leave the planetary spin unstable. Unstable spin for Venus is supported by the unusual retrograde spin of the planet which might otherwise be expected to be tidally locked.

Aerodynamic drag to the surface of Venus has been estimated by attempting to quantify the mean wind flow at the planet's surface due to super rotation (equation 11). As an upper bound to the surface wind flow generated by super rotation the measurements of local wind flow might be used. Typically wind speeds at the surface of Venus are ( $80 \%$ of the time) $>0.1 \mathrm{~m} / \mathrm{s}$ (Yamamoto 2017). Lacking a better estimate this has been taken as that due to super rotation. Classical skin friction to the surface can then be employed and compared to $g D$ torque. As can be seen in Table 2 (and Table A4) there is reasonable agreement between this estimate of aerodynamic drag to the surface of Venus and the atmospheric $g D$ torque. As can be seen employing the same crude estimation there is also seen good agreement for Titan and Mars. Earth does have a significant magnetic field and interestingly here there is reasonable (ROM) agreement between the estimates of torque due to 
Table 2. A compilation of order of magnitude estimates of; Atmospheric $g D$ torque for some of the terrestrial planets (using equation 7, Table A2) and atmospheric surface drag since these planets are essentially without a magnetosphere (equation 7, and 11, Table A4). Torques are estimated for the liquid layers of the planets (lithosphere) including $g D$ acceleration and tidal drag (equations 4 and 6 , Table A6). The tidal $(k / Q T)$ and the $g D(k / Q D)$ parameter have been taken as $10^{-2}$, see section 3.2.

\begin{tabular}{ccccc}
\hline & \multicolumn{2}{c}{ Atmospheric Torque } & \multicolumn{2}{c}{ Liquid layer Torque } \\
Body & gD torque accel. Nm & surface drag Nm & gD Torque Nm & Tidal Torque Nm \\
\hline Mars & $+4 \times 10^{13}$ & $3 \times 10^{13}$ & $+0.6 \times 10^{12}$ & $-5 \times 10^{12}$ \\
Venus & $+8 \times 10^{17}$ & $7 \times 10^{17}$ & $+2 \times 10^{12}$ & $-7 \times 10^{15}$ \\
Titan (Saturn) & $+10 \times 10^{15}$ & $4 \times 10^{15}$ & 0 & 0 \\
\hline
\end{tabular}

magnetospheric drag, atmospheric surface drag and $\mathrm{gD}$ acclererative torque. Note that in all of these cases there is observed similar super rotation.

Mercury is essentially tidally locked, has no atmosphere or magnetosphere and is therefore stable with regard to $\mathrm{gD}$.

\subsection{Liquid layer Doppler acceleration and Tidal drag}

In this section the liquid layer model is applied to most planets as well as (larger) asteroids. Here the $g D$ torque is compared to tidal drag and is of relevance to their spin orbit coupling and stability.

\subsubsection{Gas giant moons}

In Table A6 the liquid models for tidal drag and $g D$ spin acceleration (equation 4 and 6 ) are applied to the four major moons of the gas giant planets. In Table 1 the total liquid layer torques are also summarized for the liquid layers. As can be seen in all cases (Table 1 columns 6 and 7) the tidal torque on the planet is significantly (often more than an order of magnitude) greater than the $g D$ torque. In this model the lag angle ( $k / Q$ factor) is of great uncertainty. Here a value of tidal $k / Q=10^{-4}$ is mid range, though could be $10^{-3}-10^{-5}$ (Lainey 2016). For $g D$ the $k / Q D$ (lag angle) has been likely over estimated (as around 0.01).

Within uncertainties (in the $k / Q$ values) there could in fact be close balance between $g D$ and tidal torques specifically for values of $k / Q=10^{-5}$ and $k / Q D=10^{-2}$. This would in fact lead to improved stability of these spinorbit systems and therefore be further supportive of the $g D$ model. Note that in this model gravitational coupling of the moons with each other has been ignored for simplicity.

However the magnitude of atmospheric and magnetospheric torques presented in section 3.1.1 and 3.1.3 are generally 2-3 orders of magnitude greater than the torques estimated for the liquid layers of the gas giants and their moons (see Table 1). As discussed it is unclear where within the atmosphere the coupling between atmospheric $g D$ and magnetospheric drag might occur. It is possible that liquid layer torques are dominated by the balance of torques in the atmosphere or it is possible that the coupling between atmosphere and liquid layers is weak. In either case this does not constitute a failure of the $g D$ model.

\subsubsection{Earth, Venus, Mars and Titan}

The tidal drag and $g D$ spin acceleration (equation 4 and 6) have also been applied to Venus, Earth and Mars, see Table 2 and Table A6. These planets are seen to be dominated by tidal drag and therefore predicted to be (and conventionally considered) unstable with regard to the liquid layer alone.

For the earth it is well established that there is both a liquid outer core (beneath the mantle) and solid inner core. Qualitatively these might also be susceptible to a significant net tidal drag (of the liquid layer) and net $g D$ torque of the solid/plasma inner core. This might help explain the observed super rotation of the inner core (of around $0.5^{\circ} /$ year) and the sub rotation of the outer core (Mohazzabi and Skalbeck 2015). Quantitative modelling here is challenging (complex) also since magnetospheric coupling of these layers might be expected.

For Venus and Mars these planets have no magnetosphere and it has been assumed that the atmospheric $g D$ torque is balanced by surface drag (section 2.5). This implies that the entire liquid and atmospheric torque budget should be balanced for them to be stable. Mars is in fact seen to be close to being stable (balanced torques), within an order of magnitude. Venus is not predicted or seen to have stable spin. Titan is essentially tidally locked to Saturn with therefore negligible liquid layer $g D$ or tidal effects. 


\subsubsection{Asteroids}

Previous analysis has concluded that asteroids range from those that are essentially solid objects to others with high (>30\%) macro-porosity which are loosely consolidated (essentially 'rubble piles') (Britt and Consolmagno 2001; Britt et al. 2002). In the case of a poorly cohered rubble pile it would seem reasonable to apply the liquid model for such asteroids. Here balance between $g D$ torque and tidal drag leads to the prediction (using equations 4 and 6) that stability for non-tidally locked (and non-tilted) spinning bodies in a gravitational field require an angular velocity of; $w \approx c / r$ (where $r$ is the distance to the gravitating body). Specifically this would predict stable spins for the asteroids due to the Sun of order $w \approx 10^{-3}$ (i.e. around $14 \mathrm{rev}$./day). In fact a large community of asteroids do have spin periods of this order, others have extremely slow rotations (essentially tidally locked) (Pravec et al. 2002). However, for the Suns gravitational field the $g D$ or tidal torques are small leading to stabilization times $w /(d w / d t)$ of order $10^{12}$ yrs. This is long compared to the solar system age and therefore essentially inconclusive.

For the case of asteroids that are essentially solid objects, possibly the smaller community ( $\ll 1 \mathrm{~km})$, they may behave as solid rotators, see section 2.1. In this case equation 2 can be applied. At around 3 AU from the Sun this would lead to an unstable spin on a time scale of around $10^{4}$ yrs. In this case rigid bodies at this distance would be predicted to either be tidally locked (have zero relative spin) or be unstable. In fact there is observed an excess of highly (and also slow) spinning small asteroids. However it is thought that asteroid spin is dominated by a combination of collisions, tidal encounters, solar radiation effects such as the YORP effect and also be limited by their low cohesion (Rubincam 2000). Many small asteroids spin at their cohesive limit, with non-collisional asteroid breakup having been observed (asteroid P/2013 R3). Specifically the YORP effect predicts a spin-up instability of asteroids of $<$ $100 \mathrm{~m}$ in diameter also on a time scale of $<10^{4}$ years (Bottke et al. 2015). Again observations of small asteroid spin is inconclusive with regard to $g D$ effects.

Understanding asteroid belt stability and evolution remains problematic for conventional models with for example the so called mass deficit issue of the main belt (e.g. Morbidelli et al. 2015) in which it is argued that $99.9 \%$ of the asteroid belt has been lost (Pravec et al. 2002). The predicted $g D$ torque could provide an additional instability of smaller asteroids to help explain this deficit.

\section{Conclusion}

For the primary goal of critically testing the $g D$ model no conclusive observational data has been found to contradict this model within the solar system i.e. disprove the existence of a relativistic differential Doppler effect. In fact there are several cases for which this model better describes observations compared to conventional gravitation models and sheds light on otherwise unrelated and unexpected observations. Of most significance has been the prediction of atmospheric $g D$ torque (acceleration) which balances magnetospheric (drag) and quantitatively explains the internal atmospheric heat source of gas giants and the ubiquitous super rotation seen in planetary atmospheres and the Sun. These findings are summarized below;

- Atmospheric $g D$ torque quantitatively predicts super rotation/differential rotation in the Sun, Gas giants, Earth and Venus balancing torques due to magnetospheric drag and in Venus balancing the surface wind stress to the surface; Tables 1, A2, A3 and $\mathrm{A} 4$

- Atmospheric $g D$ torque quantitatively describes the internal heating of the gas giants with frictional power balance between magnetospheric drag and gD acceleration; Table 1.

- Atmospheric $g D$ torque quantitatively predicts the relatively low internal heating of Uranus (or lack of anomalous heat source) compared to Neptune (Table 1, A2, A3)

- Atmospheric $g D$ torque quantitatively provides a new mechanism for solar coronal heating through frictional power balance between magnetospheric drag and gD acceleration; Table 1, and a qualitative explanation for thermospheric heating of the gas giants as well as the relation between climate and the magnetosphere of Earth.

- Lithospheric $g D$ torque qualitatively stabilizes spinorbit coupling of planets/moons, balancing tidal torque. Quantitatively $g D$ acceleration for the liquid layers of the planets is dominated by tidal drag (by their moons and the Sun) Table 1, Table 2 and Table A6.

- Lithospheric $g D$ torque quantitatively predicts stable angular velocity of larger asteroids. Possible rigid body $g D$ torque qualitatively predicts destabilization of smaller asteroids (similar in magnitude to the YORP effect) helping explain the mass deficit and excess of high and low spinning asteroids. 
It is hoped that the work presented here stimulates further study, ideally involving experimental or observational measurement of $\mathrm{gD}$.

Acknowledgment: This project has received funding from the European Union's Horizon 2020 research and innovation program under grant agreement No 871149.

Conflict of Interest: There is no conflict of interest.

Data Availability Statement: All data generated or analyzed during this study are included in this published article.

\section{References}

Aschwanden MJ. 2001. An Evaluation of Coronal Heating Models for Active Regions Based on Yohkoh, SOHO, and TRACE Observations. Astrophys J. 560(2):1035-1044.

Aschwanden MJ. 2005. Physics of the Solar Corona: An Introduction with Problems and Solutions. Chichester, UK: Praxis Publishing.

Bottke WF, Brož M, O’Brien DP, Campo Bagatin A, Morbidelli A, Marchi S. 2015. The collisional evolution of the main asteroid belt. In: Michel P, et al., editors. Asteroids IV. Tucson: Univ. of Arizona; p. 701-724.

Britt DT, Consolmagno GJ. 2001. Asteroid bulk density: Implications for the structure of asteroids. Proceedings of the 32nd Annual Lunar and Planetary Science Conference, March 12-16, Houston, Texas.

Britt DT, Yeomans D, Housen K, Consolmagno GJ. 2002. Asteroid density, porosity, and structure. In: Bottke WF, editor. Asteroids III. University of Arizona Press; p. 485.

Chanover N. 2013. Atmospheres of Jovian Planets. In: Oswalt TD, French LM, Kalas P, editors. Planets, Stars and Stellar Systems. Dordrecht: Springer.

Courtillot V, Gallet Y, Le Mouël JL, Fluteau F, Genevey A. 2007. Are there connections between the Earth's magnetic field and climate? Earth Planet Sci Lett. 253(3-4):328-339.

Cowley SWH, Nichols JD, Jackman CM. 2015. Down-tail mass loss by plasmoids in Jupiter's and Saturn's magnetospheres. J Geophys Res Space Phys. 120(8):6347-6356.

Goldreich P, Soter S. 1966. Q in the Solar System. Icarus. 5(1-6):375389.

Hanel RA, Conrath BJ, Kunde VG, Pearl JC, Pirraglia JA. 1983. Albedo, internal heat flux, and energy balance of Saturn. Icarus. 53(2):262-285.

Helled R, Anderson JD, Podolak M, Schubert G. 2011. Interior models of Uranus and Neptune. Astrophys. J. 726:15.

Holmberg MKG, Wahlund J-E, Vigren E, Cassidy TA, Andrews DJ. 2016. Transport and chemical loss rates in Saturn's inner plasma disk. J Geophys Res Space Phys. 121(3):2321-2334.

Hunten DM, Veverka J. 1976. Stellar and spacecraft occultation by Jupiter: A critical review of derived temperature profiles. In:
Gehrels T. Editor. Jupiter, University of Arizona Press, Tucson, p. 247-283.

Ishikawa S, Glesener L, Krucker S, Christe S, Buitrago-Casas JC, Narukage N, et al. 2017. Detection of nanoflare-heated plasma in the solar corona by the FOXSI- 2 sounding rocket. Nat Astron. 1(11):771-774.

Klimchuk JA. 2006. On solving the coronal heating problem. Sol Phys. 234(1):41-77.

Kong D, Zhang K, Schubert G, Anderson JD. 2018. Origin of Jupiter's cloud-level zonal winds remains a puzzle even after Juno. Proc Natl Acad Sci USA. 115(34):8499-8504.

Kumar N, Kumar P, Singh S. 2006. Coronal heating by MHD waves. Astron Astrophys. 453(3):1067-1078.

Kundt W. No. I I. Vol. 31. Pergamon Press; 1983. p. 1339-1343. (ATMOSPHERIC SUPERROTATION ON SATURN AND JUPITER, Planet. Spore SW).

Lainey V. 2016. Quantification of tidal parameters from Solar System data. Celestial Mech Dyn Astron. 126(1-3):145-156.

Li L, Jiang X, West RA, Gierasch PJ, Perez-Hoyos S, Sanchez-Lavega A, et al. 2018. Less absorbed solar energy and more internal heat for Jupiter. Nat Commun. 9(1):3709.

Lewis SR, Dawson J, Read PL, Mendonça J, Ruan T, Montabone L. 2012. Super-rotating jets in the atmospheres of terrestrial planets. Comparative Climatology of Terrestrial Planets, 8051.

Lund MN, Miesch MS, Christensen-Dalsgaard J. 2014. Differential rotation in main-sequence solar-like stars: Qualitative inference from asteroseismic data. The Astrophys. J. 790:121.

Macdonald GJF. 1964. Tidal Friction. Rev Geophys. 2(3):467.

Merrison JP. 2016. A Modified Relativistic Model of Gravitational Propagation. IJAA. 6(3):312-327.

Miesch MS, Toomre J. 2009. Turbulence, Magnetism, and Shear in Stellar Interiors. Annu Rev Fluid Mech. 41(1):317-345.

Mlynczak MG, Hunt LA, Russell JM 3rd, Marshall BT, Mertens CJ, Thompson RE. 2016. The global infrared energy budget of the thermosphere from 1947 to 2016 and implications for solar variability. Geophys Res Lett. 43(23):11934-11940.

Modisette JL. 1967. Solar wind induced torque on the Sun. J Geophys Res. 72(5):1521-1526.

Mohazzabi P, Skalbeck JD. 2015. Superrotation of Earth's inner core, Extraterrestrial Impacts, and the effective viscosity of outer core. Geophys. J. Int. 2015:763716. DOI: https://doi.org/10.1155/2015/763716.

Morbidelli A, Walsh KJ, O’Brien DP, Minton DA, Bottke WF. 2015. The dynamical evolution of the asteroid belt. In: Michel $P$, et al.. Editors. Asteroids IV. Tucson: Univ. of Arizona; p. 493-507.

O’Donoghue J, Moore L, Connerney J, Melin H, Stallard TS, Miller S. 2019. Observations of the chemical and thermal response of 'ring rain' on Saturn's ionosphere. Icarus. 322:251-260.

Pandey K, Narain U. 2001. On solar coronal heating. Bull Astron Soc India. 29:231-238.

Pravec P, Harris AW, Michalowski T. 2002. Asteroid rotations. In: Bottke WF, Cellino A, Paolicchi P, Binzel RP. Editors. Asteroids III. Tucson: Univ. of Arizona; p. 113-122.

Read PL, Lebonnois S. 2018. Superrotation on Venus, on Titan, and Elsewhere. Annu Rev Earth Planet Sci. 46(1):175-202.

Richardson JD, Belcher JW, Lazarus AJ, Paularena KI, Gazis PR. 1996. Statistical properties of the solar wind Citation. AIP Conf Proc. 382:483-486.

Rishbeth H. 1971. Rotation of the variation of upper atmosphere. Nature. 229(5283): 333-334. 
Rubincam DP. 2000. Radiative spin-up and spin-down of small asteroids. Icarus. 148(1):2-11.

Schneider T, Liu J. 2009. Formation of Jets and Equatorial Superrotation on Jupiter. J Atmos Sci. 66(3):579-601.

Siscoe GL. 2001. 70 years of magnetospheric modeling, space weather. Geophysical Monograph 125 by the American Geophysical Union.

Suess S. 1999. Overview and current knowledge of the solar wind and the corona. The Solar Probe. NASA/Marshall Space Flight Center.

Yamamoto M. 2017. Probability distribution of surface wind speed induced by convective adjustment on Venus. Icarus. 284:314324.
Yung YL, Lyons JR, Selesnick RS. 1990. Heating of Neptunes thermosphere by the magnetospheric electric field. Abstract of the 22nd Annual DPS. Id. 1105.

Zhu X. 2005. Dynamics in planetary atmospheric physics: Comparative studies of equatorial superrotation for Venus, Titan, and Earth. Johns Hopkins, APL Technical Digest. 26(2):164-174.

Zhu X. 2006. Maintenance of equatorial superrotation in the atmospheres of Venus and Titan. Planet Space Sci. 54(8):761-773. 


\section{Appendix}

Table A1. input parameters for this modelling work.

\begin{tabular}{ccccccccc}
\hline Planet & $\begin{array}{c}\text { Mass } \\
\mathbf{M ~ k g}\end{array}$ & $\begin{array}{c}\text { Radius } \\
\mathbf{R ~ M}\end{array}$ & $\begin{array}{c}\text { Orbital radius } \\
\mathbf{r ~ m}\end{array}$ & $\begin{array}{c}\text { Period } \\
\text { T Hrs }\end{array}$ & $\begin{array}{c}\text { Angular velocity } \\
\text { wo s-1 }\end{array}$ & $\begin{array}{c}\text { Surface velocity } \\
\text { Vrot m/s } \mathbf{s}\end{array}$ & $\begin{array}{c}\text { Gravitational } \\
\text { acceleration } \\
\text { go m/s2 }\end{array}$ & $\begin{array}{c}\text { Spin Tilt } \\
\circ\end{array}$ \\
\hline Jupiter & $1.9 \times 10^{27}$ & $7.1 \times 10^{7}$ & $7.8 \times 10^{11}$ & 9.9 & $1.8 \times 10^{-4}$ & 12511 & $2.2 \times 10^{-4}$ \\
Saturn & $5.7 \times 10^{26}$ & $6.0 \times 10^{7}$ & $1.4 \times 10^{12}$ & 10.2 & $1.7 \times 10^{-4}$ & 10261 & $6.8 \times 10^{-5}$ & 3.1 \\
Uranus & $8.7 \times 10^{25}$ & $2.4 \times 10^{7}$ & $2.9 \times 10^{12}$ & 10.7 & $1.6 \times 10^{-4}$ & 3912 & $1.6 \times 10^{-5}$ \\
Neptune & $1.0 \times 10^{26}$ & $2.2 \times 10^{7}$ & $4.5 \times 10^{12}$ & 15.8 & $1.1 \times 10^{-4}$ & 2429 & $6.6 \times 10^{-6}$ & 28 \\
Sun & $2.0 \times 10^{30}$ & $7.0 \times 10^{8}$ & $7.8 \times 10^{11}$ & 600 & $2.9 \times 10^{-6}$ & 2024 & $2.1 \times 10^{-7}$ & 0 \\
Earth & $6.0 \times 10^{24}$ & $6.4 \times 10^{6}$ & $1.5 \times 10^{11}$ & 24 & $7.3 \times 10^{-5}$ & 465 & $6.0 \times 10^{-3}$ & 23 \\
Venus & $4.8 \times 10^{24}$ & $6.1 \times 10^{6}$ & $1.1 \times 10^{11}$ & 5832 & $3.0 \times 10^{-7}$ & 1.8 & $1.2 \times 10^{-2}$ & 3.9 \\
Mars & $6.4 \times 10^{23}$ & $3.4 \times 10^{6}$ & $2.3 \times 10^{11}$ & 24.6 & $7.1 \times 10^{-5}$ & 241 & $2.5 \times 10^{-3}$ & 1.8 \\
\hline
\end{tabular}

Table A2. Order of magnitude estimates of 'atmospheric' $g D$ torque (equation 7) and power generation (equation 12) for the gas giants, some terrestrial planets/moon and the Sun (plamasphere).

\begin{tabular}{|c|c|c|c|c|c|}
\hline Body & $\begin{array}{c}\text { Atmospheric thickness } \\
M\end{array}$ & $\begin{array}{l}\text { Atmospheric density average } \\
\qquad \mathrm{kg} / \mathrm{m} 3\end{array}$ & $\begin{array}{l}\text { Atmospheric effective mass } \\
\mathrm{kg}\end{array}$ & $\begin{array}{l}\text { Torque gD } \\
\quad \mathrm{Nm}\end{array}$ & $\begin{array}{l}\text { Power gD } \\
\text { W }\end{array}$ \\
\hline Jupiter & $1 \times 10^{5}$ & 0.4 & $2.5 \times 10^{21}$ & $8.0 \times 10^{20}$ & $1.4 \times 10^{17}$ \\
\hline Saturn & $1 \times 10^{5}$ & 0.4 & $1.8 \times 10^{21}$ & $8.9 \times 10^{19}$ & $1.5 \times 10^{16}$ \\
\hline Uranus & $1 \times 10^{6}$ & 0.4 & $2.9 \times 10^{21}$ & $4.5 \times 10^{17}$ & $7.3 \times 10^{13}$ \\
\hline Neptune & $1 \times 10^{6}$ & 0.4 & $2.4 \times 10^{21}$ & $2.7 \times 10^{18}$ & $3.0 \times 10^{14}$ \\
\hline Sun & $1 \times 10^{8}$ & 10 & $6.0 \times 10^{27}$ & $2.4 \times 10^{24}$ & $6.9 \times 10^{18}$ \\
\hline \multicolumn{6}{|l|}{ (g Jupiter) } \\
\hline Earth & $1 \times 10^{4}$ & 1 & $5.1 \times 10^{18}$ & $1.1 \times 10^{17}$ & $8.1 \times 10^{12}$ \\
\hline Venus & $1 \times 10^{5}$ & 10 & $4.6 \times 10^{20}$ & $7.6 \times 10^{17}$ & $2.2 \times 10^{12}$ \\
\hline Mars & $1 \times 10^{4}$ & 0.01 & $1.4 \times 10^{16}$ & $4.0 \times 10^{13}$ & $2.8 \times 10^{9}$ \\
\hline Titan & $1 \times 10^{4}$ & 4.7 & $3.9 \times 10^{18}$ & $9.5 \times 10^{15}$ & $6.5 \times 10^{10}$ \\
\hline
\end{tabular}

Table A3. Order of magnitude estimates of magnetospheric drag (equation 8) due to solar wind pressure which has been taken as around on average $6 \mathrm{nPa}$ at $1 \mathrm{AU}$ from the Sun, for the other solar system bodies the solar wind pressure has been estimated to vary as $1 / \mathrm{r}^{2}$ with $\mathrm{r}$ the distance to the Sun. For the Sun the measured internal power refers to the coronal heating power required (in a quiet region/period).

\begin{tabular}{cccccccc}
\hline Body & $\begin{array}{c}\text { Solar wind pressure } \\
P=n m v B 2 \mathrm{~Pa}\end{array}$ & $\begin{array}{c}\text { Solar wind velocity } \\
\text { +Bfield velocity vB } \\
\mathrm{m} / \mathrm{s}\end{array}$ & $\begin{array}{c}\text { Bow shock } \\
\text { radius } \\
\text { RB.Rp }\end{array}$ & $\begin{array}{c}\text { Area Bow } \\
\text { shock A } \\
\mathrm{m}^{2}\end{array}$ & $\begin{array}{c}\text { B Torque } \\
\text { P.A.RB.Rp } \\
\text { Nm }\end{array}$ & $\begin{array}{c}\text { Power B } \\
\text { P.A.vB } \\
\text { W }\end{array}$ & $\begin{array}{c}\text { Measured } \\
\text { internal heating } \\
\text { W }\end{array}$ \\
\hline Jupiter & $2.7 \times 10^{-9}$ & $1.7 \times 10^{6}$ & 100 & $1.6 \times 10^{20}$ & $3.1 \times 10^{21}$ & $7.5 \times 10^{17}$ & $1.1 \times 10^{17}$ \\
Saturn & $1.7 \times 10^{-10}$ & $7.8 \times 10^{5}$ & 27 & $8.2 \times 10^{18}$ & $2.2 \times 10^{18}$ & $1.1 \times 10^{15}$ & $2.3 \times 10^{16}$ \\
Uranus & $2.2 \times 10^{-11}$ & $5.9 \times 10^{5}$ & 23 & $9.6 \times 10^{17}$ & $1.2 \times 10^{16}$ & $1.3 \times 10^{13}$ & $7.6 \times 10^{13}$ \\
Neptune & $9.1 \times 10^{-12}$ & $5.9 \times 10^{5}$ & 35 & $1.9 \times 10^{18}$ & $1.3 \times 10^{16}$ & $1.0 \times 10^{13}$ & $6.1 \times 10^{14}$ \\
Sun & $2.8 \times 10^{-4}$ & $5 \times 10^{5}$ & 1 & $6.0 \times 10^{18}$ & $1.2 \times 10^{24}$ & $3.4 \times 10^{19}$ & $(\mathrm{co}) 6 \times 10^{19}$ \\
Earth & $6 \times 10^{-9}$ & $5.1 \times 10^{5}$ & 20 & $5.1 \times 10^{16}$ & $4.0 \times 10^{16}$ & $1.6 \times 10^{14}$ & $\left(\right.$ th) $1 \times 10^{12}$ \\
\hline
\end{tabular}


Table A4. Order of magnitude estimates of atmospheric-surface drag for terrestrial planets/moons with atmospheres. For Venus the effective surface velocity due to super rotation has been taken as the minimum average observed surface wind speed, specifically for $80 \%$ of the time winds have been seen $>0.1 \mathrm{~m} / \mathrm{s}$ (this includes other sources of wind generation e.g. solar) so possibly an over estimate. In the upper atmosphere of all of these planets/moons the super rotation wind speeds are of order $100 \mathrm{~m} / \mathrm{s}$. The effective surface velocity due to super rotation for Earth, Mars and Titan have also been taken as $0.1 \mathrm{~m} / \mathrm{s}$ lacking better values.

\begin{tabular}{lcccccc}
\hline Body & $\begin{array}{c}\text { Atmospheric mean } \\
\text { density rho } \\
\mathrm{Kg} / \mathrm{m}^{3}\end{array}$ & $\begin{array}{c}\text { Surface velocity } \\
\text { effective } \mathrm{m} \\
\mathrm{m} / \mathrm{s}\end{array}$ & $\begin{array}{c}R p \\
\mathrm{~m}\end{array}$ & $\begin{array}{c}\text { Surface Area } \\
0.4 .4 . p i . R p 2 \\
\mathrm{~m}^{2}\end{array}$ & $\begin{array}{c}\text { Drag force } \\
F=\text { cd.rho.u2.A }\end{array}$ & $\begin{array}{c}\text { Torque atmospheric } \\
\text { drag } F . R p \\
\mathrm{Nm}\end{array}$ \\
\hline Venus & 67 & 0.1 & $6.1 \times 10^{6}$ & $1.8 \times 10^{14}$ & $1.2 \times 10^{11}$ & $7.3 \times 10^{17}$ \\
Earth & 1 & 0.1 & $6.4 \times 10^{6}$ & $2.0 \times 10^{14}$ & $2.0 \times 10^{9}$ & $1.3 \times 10^{16}$ \\
Mars & 0.02 & 0.1 & $3.4 \times 10^{6}$ & $5.2 \times 10^{13}$ & $1.1 \times 10^{7}$ & $3.4 \times 10^{13}$ \\
Titan & 4.7 & 0.1 & $2.6 \times 10^{6}$ & $3.2 \times 10^{13}$ & $1.5 \times 10^{9}$ & $4.0 \times 10^{15}$ \\
\hline
\end{tabular}

Table A5. Order of magnitude estimates of the Tidal gradient in $g$ field $\left(d g T=G M R / r^{3}\right)$ ) across the body compared to the $d g D$ gradient $\left(d g D=G M R / r^{2} \cdot v / c\right)$ for planets and the Sun due to the major satellites (and the Sun, see Table A7), see section 2.2. In all cases the total $d g T$ is more than $\times 10$ greater than $d g D$ (dominated typically by moons). This implies generally that tidal distortions dominate over $g D$ distortions.

\begin{tabular}{|c|c|c|c|c|c|c|}
\hline \multicolumn{7}{|c|}{ Tidal $g(d g / d r)=G m R / r 3$} \\
\hline Body & Moon 1 & Moon 2 & Moon 3 & Moon 4 & Sun & Total \\
\hline Jupiter & $5.7 \times 10^{-6}$ & $7.6 \times 10^{-7}$ & $5.8 \times 10^{-7}$ & $7.7 \times 10^{-8}$ & $2.1 \times 10^{-8}$ & $7.2 \times 10^{-6}$ \\
\hline Saturn & $8.3 \times 10^{-8}$ & $6.3 \times 10^{-8}$ & $3.0 \times 10^{-7}$ & $1.6 \times 10^{-10}$ & $2.9 \times 10^{-9}$ & $4.5 \times 10^{-7}$ \\
\hline Uranus & $3.2 \times 10^{-7}$ & $1.0 \times 10^{-7}$ & $6.9 \times 10^{-8}$ & $2.5 \times 10^{-8}$ & $1.5 \times 10^{-10}$ & $5.1 \times 10^{-7}$ \\
\hline Neptune & $1.8 \times 10^{-8}$ & $4.6 \times 10^{-8}$ & $7.2 \times 10^{-7}$ & $2.4 \times 10^{-13}$ & $3.2 \times 10^{-11}$ & $7.8 \times 10^{-7}$ \\
\hline Sun & $1.9 \times 10^{-10}$ & $9.7 \times 10^{-12}$ & $1.8 \times 10^{-10}$ & $8.3 \times 10^{-11}$ & NA & $4.5 \times 10^{-10}$ \\
\hline Earth & $5.8 \times 10^{-7}$ & NA & NA & NA & $2.5 \times 10^{-7}$ & $8.3 \times 10^{-7}$ \\
\hline Venus & NA & NA & NA & NA & $6.5 \times 10^{-7}$ & $6.5 \times 10^{-7}$ \\
\hline Mars & $3.0 \times 10^{-9}$ & $3.7 \times 10^{-11}$ & NA & NA & $4.3 \times 10^{-8}$ & $4.6 \times 10^{-8}$ \\
\hline \multicolumn{7}{|c|}{$g D=G m R / r 2 . v / c$} \\
\hline Body & Moon 1 & Moon 2 & Moon 3 & Moon 4 & Sun & Total \\
\hline Jupiter & $1.1 \times 10^{-9}$ & $2.6 \times 10^{-10}$ & $3.4 \times 10^{-10}$ & $8.3 \times 10^{-11}$ & $9.4 \times 10^{-9}$ & $1.1 \times 10^{-8}$ \\
\hline Saturn & $1.5 \times 10^{-11}$ & $1.7 \times 10^{-11}$ & $2.0 \times 10^{-10}$ & $3.2 \times 10^{-13}$ & $2.3 \times 10^{-9}$ & $2.6 \times 10^{-9}$ \\
\hline Uranus & $2.7 \times 10^{-11}$ & $1.3 \times 10^{-11}$ & $1.5 \times 10^{-11}$ & $7.5 \times 10^{-12}$ & $2.2 \times 10^{-10}$ & $2.9 \times 10^{-10}$ \\
\hline Neptune & $9.8 \times 10^{-14}$ & $8.0 \times 10^{-13}$ & $1.0 \times 10^{-10}$ & $4.8 \times 10^{-16}$ & $5.4 \times 10^{-11}$ & $1.6 \times 10^{-10}$ \\
\hline Sun & $1.4 \times 10^{-12}$ & $1.3 \times 10^{-13}$ & $1.7 \times 10^{-13}$ & $1.1 \times 10^{-13}$ & NA & $1.8 \times 10^{-12}$ \\
\hline Earth & $5.3 \times 10^{-11}$ & NA & NA & NA & $9.2 \times 10^{-9}$ & $9.3 \times 10^{-9}$ \\
\hline Venus & NA & NA & NA & NA & $1.5 \times 10^{-10}$ & $1.5 \times 10^{-10}$ \\
\hline Mars & $2.1 \times 10^{-14}$ & $1.7 \times 10^{-16}$ & NA & NA & $2.3 \times 10^{-9}$ & $2.3 \times 10^{-9}$ \\
\hline
\end{tabular}


Table A6. Order of magnitude estimates of $g D$ and tidal torque for the liquid layers of the planets due to major satellites (see Table A7) using equations 4 and 6 . Note that Mercury is essentially tidally locked. Here the empirical parameters $(k / Q)$ and $(k / Q D)$ have been taken as 0.01 for the terrestrial planets. For the gas giants $(k / Q D)$ has been taken as 0.01 (as an upper estimate) and the $k / Q=10^{-4}$ (see section 2.2 and 3.2).

\begin{tabular}{|c|c|c|c|c|c|c|}
\hline \multicolumn{7}{|c|}{ TIDAL TORQUE LIQUID } \\
\hline Body & Moon 1 & Moon 2 & Moon 3 & Moon 4 & Sun & Total \\
\hline Jupiter & $8.7 \times 10^{18}$ & $1.5 \times 10^{17}$ & $8.8 \times 10^{16}$ & $1.6 \times 10^{15}$ & $1.2 \times 10^{14}$ & $9.0 \times 10^{18}$ \\
\hline Saturn & $1.1 \times 10^{15}$ & $6.4 \times 10^{14}$ & $1.4 \times 10^{16}$ & $4.2 \times 10^{9}$ & $1.4 \times 10^{12}$ & $1.6 \times 10^{16}$ \\
\hline Uranus & $1.0 \times 10^{15}$ & $1.0 \times 10^{14}$ & $4.8 \times 10^{13}$ & $6.3 \times 10^{12}$ & $2.2 \times 10^{8}$ & $1.2 \times 10^{15}$ \\
\hline Neptune & $2.7 \times 10^{12}$ & $1.7 \times 10^{13}$ & $4.1 \times 10^{15}$ & $4.6 \times 10^{2}$ & $8.3 \times 10^{6}$ & $4.2 \times 10^{15}$ \\
\hline Mars & $2.7 \times 10^{10}$ & - & NA & NA & $5.4 \times 10^{12}$ & $5.4 \times 10^{12}$ \\
\hline Earth & $6.5 \times 10^{15}$ & NA & NA & NA & $1.3 \times 10^{15}$ & $7.8 \times 10^{15}$ \\
\hline Venus & NA & NA & NA & NA & $7.1 \times 10^{15}$ & $7.1 \times 10^{15}$ \\
\hline \multicolumn{7}{|c|}{$g D$ TORQUE LIQUID } \\
\hline Body & Moon 1 & Moon 2 & Moon 3 & Moon 4 & Sun & Total \\
\hline Jupiter & $4.3 \times 10^{17}$ & $1.2 \times 10^{16}$ & $1.1 \times 10^{16}$ & $3.5 \times 10^{14}$ & $1.1 \times 10^{16}$ & $4.6 \times 10^{17}$ \\
\hline Saturn & $4.7 \times 10^{13}$ & $3.9 \times 10^{13}$ & $2.0 \times 10^{15}$ & $1.7 \times 10^{9}$ & $2.2 \times 10^{14}$ & $2.3 \times 10^{15}$ \\
\hline Uranus & $2.1 \times 10^{13}$ & $3.0 \times 10^{12}$ & $2.3 \times 10^{12}$ & $4.0 \times 10^{11}$ & $6.7 \times 10^{10}$ & $2.7 \times 10^{13}$ \\
\hline Neptune & $1.5 \times 10^{10}$ & $1.5 \times 10^{11}$ & $1.1 \times 10^{14}$ & $1.8 \times 10^{2}$ & $2.8 \times 10^{9}$ & $1.1 \times 10^{14}$ \\
\hline Mars & $1.2 \times 10^{5}$ & - & NA & NA & $5.6 \times 10^{11}$ & $5.6 \times 10^{11}$ \\
\hline Earth & $1.2 \times 10^{12}$ & NA & NA & NA & $9.2 \times 10^{13}$ & $9.3 \times 10^{13}$ \\
\hline Venus & NA & NA & NA & NA & $1.5 \times 10^{12}$ & $1.5 \times 10^{12}$ \\
\hline
\end{tabular}

Table A7. List of major satellites.

\begin{tabular}{ccccc}
\hline Body & Moon 1 & Moon 2 & Moon 3 & Moon 4 \\
\hline Jupiter & Io & Europa & Ganymede & Callisto \\
Saturn & Dione & Rhea & Titan & Lapetas \\
Uranus & Ariel & Umbriel & Titania & Oberon \\
Neptune & Larissa & Proteus & Triton & Nerid \\
Sun & Jupiter & Saturn & Earth & Venus \\
Earth & Moon & NA & NA & NA \\
Venus & NA & NA & NA & NA \\
Mars & Phobos & Deimos & NA & NA \\
\hline
\end{tabular}

